\title{
Project Apeiro - High Altitude Balloon Payload for Measuring Cosmic Ray Flux
}

\section{Sanket Deshpande*}

Birla Institute of Technology and Science, Pilani

E-mail: sanket@ieee.org

\section{Lucky Kapoor}

Birla Institute of Technology and Science, Pilani

E-mail: lucky.kapoor_001@tifr.res.in

\section{Shivangi Kamat ${ }^{\dagger}$}

Birla Institute of Technology and Science, Pilani

E-mail: skamat2@wisc.edu

\section{Dipankar Pal}

Birla Institute of Technology and Science, Pilani

E-mail: dipankarp@goa.bits-pilani.ac.in

\section{Satyanarayana Bheesette}

Tata Institute of Fundamental Research, Mumbai

E-mail: bsnetifr.res.in

\begin{abstract}
The experiment (Project Apeiro) aims to determine cosmic ray flux in the lower stratospheric regions of the atmosphere by deploying a measurement instrument on a High-Altitude Balloon platform. This paper illustrates the design and construction of the experimental equipment and also highlights the precautions and tests undertaken to qualify the instrument for a near-space flight. Cosmic ray flux is measured by using detectors constructed with an optically coupled combination of scintillator blocks and photo-multiplier tubes. The signal from the detectors is recorded with a high speed data acquisition system (DAQ). The flight computer acquires data from the DAQ and other sensors and stores them on a memory. The flight data is also continuously transmitted to the ground station over a telemetry channel. The flight was conducted from Hyderabad, India on 2nd February 2019. Preliminary analysis of the flight indicates nominal operation of all equipment, and has been presented in this paper.
\end{abstract}

36th International Cosmic Ray Conference -ICRC2019

July 24th - August 1st, 2019

Madison, WI, U.S.A.

\footnotetext{
*Speaker.

${ }^{\dagger}$ Speaker.
} 


\section{Introduction}

Cosmic rays consist of charged particles which are continuously incident upon earth. While a majority of the charged particles are deflected by the magnetic field of earth, some of them are able to pierce through the magnetic field. When they collide with the molecules in the upper atmosphere of the earth, they produce lighter and short-lived particles such as pions, kaons etc, some of which further decay to produce muons, neutrinos, photons and electrons [1]. Such interactions lead to drastic loss in the intensities of these particles, thereby dampening their effect at lower altitudes.

Radiation from cosmic rays is known to cause various anomalies in the electronic devices [2] [3] [4]. Ionizing particles of cosmic ray showers are known to mutate and damage cells in human tissues [5] [6]. Astronauts and aviation crews are continually exposed to the high energy cosmic rays in space and also at high altitudes. Detailed study and analyses is required to understand the implications of cosmic ray exposure to commercial and military aircraft crew and passengers [7]. The experiment, titled Project Apeiro [8], aimed to conduct measurement and analyses of the cosmic ray flux in lower stratospheric region of earth's atmosphere by deploying an experimental setup as a payload on a High Altitude Balloon (HAB) platform.

The experiment was designed to measure cosmic ray dose rates by stacking three cosmic ray detectors vertically in the payload and obtaining a coincidence measurement from the detectors. Within the dimensions of the experimental setup, the cosmic ray particles are assumed to travel in straight lines, ignoring any curvature in its path. The general zenith angle distribution of incident cosmic rays on the earth can be denoted by:

$$
I=I_{o} \cos ^{2} \theta
$$

where $\theta$ denotes the zenith angle of the cosmic ray particle path. Hence, cosmic rays can be presumed to be nearly perpendicular to the earth's surface (with respect to the detector's dimensions) when they are incident upon earth's surface. Therefore, a cosmic ray particle will trigger all three detectors (almost) simultaneously, and this event is recorded as signal. Events wherein simultaneous detection is not recorded are rejected as noise.

This paper presents the design of an air-borne, complete cosmic-ray-detection-system payload, the results of the flight qualification tests and preliminary results from the high-altitude flight. The rest of the paper is structured as follows. Section 2 presents the cosmic ray telescope description. Section 3 describes the data acquisition system for the telescope. In Section 4, the power supply systems are described while Section 5 illustrates the protective measures and qualification and calibration tests conducted on the payload. Primary analysis of high altitude balloon (HAB)-flight data is also presented here. Section 6 concludes the paper.

\section{Cosmic Ray Telescope}

\subsection{Detector}

Each cosmic ray particle detector has been constructed by connecting a Photomultiplier Tube (PMT) - Hamamatsu R6233 - to a plastic scintillator block of 100mm X 100mm using optical glue that maintains a consistent refractive index across the coupling. The PMT anode is connected to 
a high voltage supply and the cathode is connected to the high voltage ground. The high voltage ground is kept isolated. The PMT signal is obtained from the anode via a coupling HV capacitor. The entire assembly is covered with a thick sheet of Tyvek (a light reflecting sheet) and Tedler to prevent stray light from entering the sensitive detector. The detector assembly is then inserted inside an aluminum enclosure to protect it from electromagnetic interference.

\subsection{Telescope Arrangement}

Three detectors have been constructed as stated in the previous section, and stacked vertically one above the other, in order to obtain coincidence of signal by all three detectors. This geometrical arrangement provides an aperture of $46.8^{\circ}$ and can detect over $49 \%$ of the incident cosmic ray particles [distribution modeled using equation 1.1]. The mechanical drawing of the arrangement is shown in the figure 1a, while figure $1 \mathrm{~b}$ is an actual photograph of the cosmic ray telescope. Figure $1 \mathrm{c}$ is a schematic that explains how a cosmic ray particle passes through all the detectors (generates signal) while an in-situ charged particle passes through a single detector (generates noise). Analog signals from each of the three scintillation detectors are sent to the data acquisition system for processing.

(a)

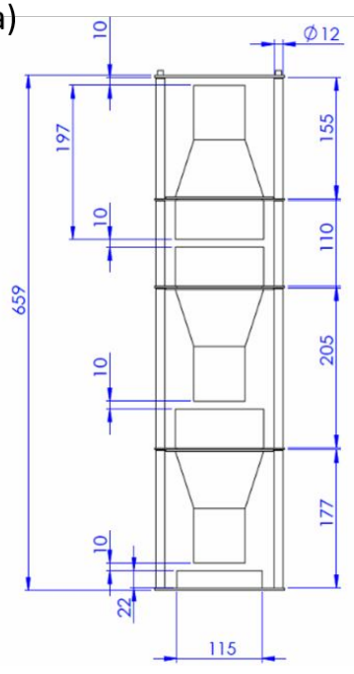

(b)

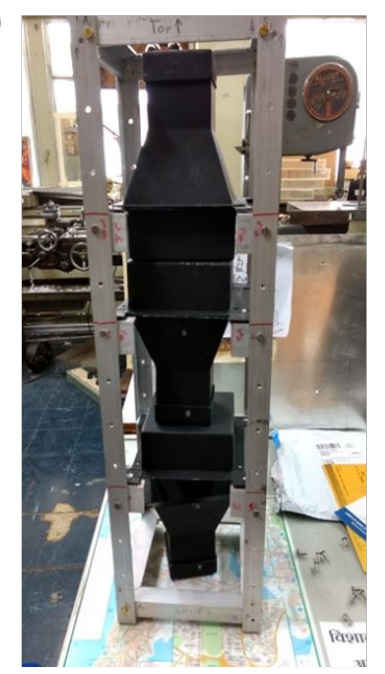

(c)

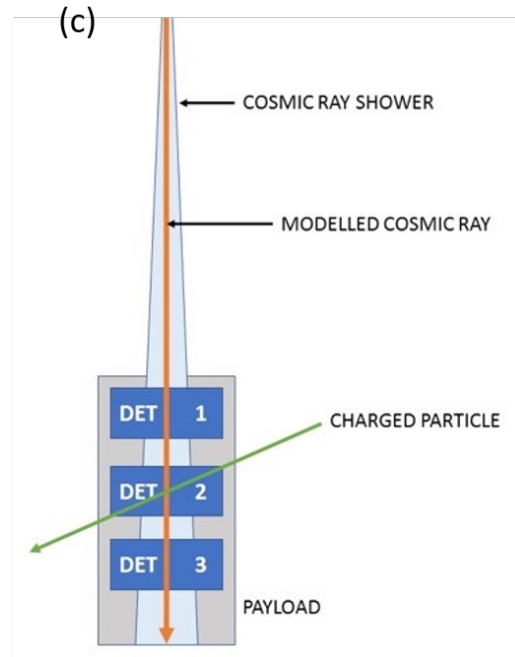

Figure 1: (a) Mechanical drawing of the cosmic ray telescope and (b) Picture of the cosmic ray telescope (c) Illustration of the arrangement of the detectors, the cosmic ray shower propagation and noise generated by in-situ charged particles

\section{Data Acquisition System}

The data acquisition system (DAQ) converts negative polarity analog signals into logic signals using leading edge discriminators and also counts their individual rates. It performs logical coincidence of signals from all three detectors and then counts them. As stated, the coincidence-signals indicate passage of comic ray particles through the apparatus. The entire data acquisition system, which includes discrimination, coincidence and counting operations is implemented on a single 
printed circuit board (PCB). The DAQ system operations are controlled by a micro-controller. Figure 2 shows a functional blocks of the data acquisition system.

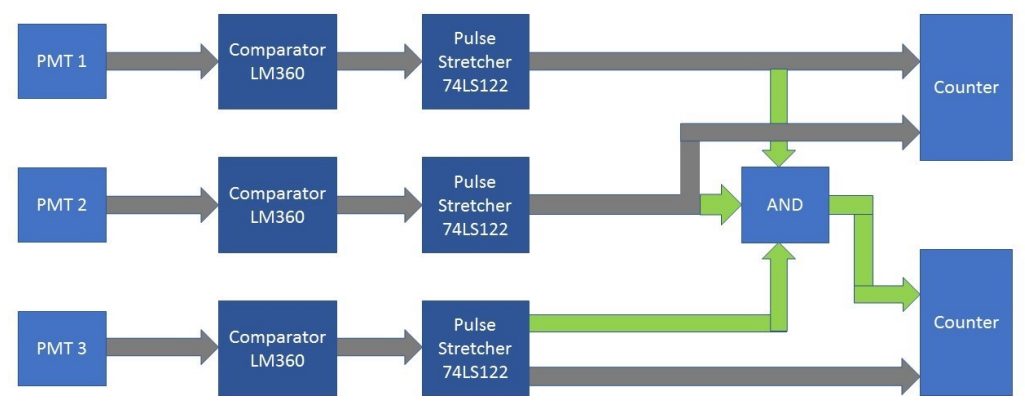

Figure 2: Functional block diagram of the data acquisition system

\subsection{Discrimination}

The first step towards processing the incoming analog signals from the PMTs (fast pulses of negative polarity) is to convert them into logic signals. Input from each PMT is passed to a high frequency differential comparator comparator- LM360. It generates a TTL-pulse of duration equal to the time period for which the analog pulse's magnitude is larger than the threshold, thereby filtering out the noise. The TTL pulse is then sent to a multivibrator- SN54LS122J, to shape each TTL-pulse to a pulse of fixed duration for all channels. The time-duration is set such that it is greater than the maximum recorded time duration of the detector in a calibration experiment, yet smaller than the average time difference between two consecutive pulses from the same detector. These signals are then individually sent to the counter ICs which count the number of signals of each detector over a uniform time interval. Since the experiment implements a three detector system, three separate channels have been designed for this purpose.

\subsection{Coincidence}

The coincidence operation, also referred as the AND operation is to identify the signal, i.e., simultaneous signal from the detectors. A select logic allows the experimenter to determine the channels that are included for the AND operation- thereby allowing for a three-fold or a two-fold coincidence. In normal operation, it is desired to include all three channels.

\subsection{Counting}

The number of pulses generated from each of the multivibrators is detected and counted over a time period of 1 second. Therefore, four different counts are measured: channel 1, channel 2, channel 3 and the coincidence channel.

A combination of SN74LV8154 counter IC coupled with SN74LVC138A demultiplexer IC has been used. The SN74LV8154 is a dual-counter IC with inbuilt register, hence two such assemblies of counter-demultiplexer are required to perform counting operation on four signals. The IC has two 16-bit counters, whose output can be read over eight output lines. A select logic is implemented using the demultiplexer to obtain the upper eight bits or lower eight bits on the output lines. 


\subsection{Flight Computer, Sensors and Data Handling}

The experiment uses the MSP430F5438 Experimenter Board from TI as its flight computer. It acquires the following data: counting rates from all the channels on the DAQ system, internal temperature and pressure (BMP180 sensor), polar and azimuthal angular inclinations (MPU-9250 IMU module), GPS information (Garmin 18x LVC module). Following the acquisition, it stores the data in an onboard EEPROM (Spansion S25FL216K 16MB) and transmits it over the telemetry system to the ground station. It receives commands as interrupts from the ground station, if any, over a buffered tele-command channel.

On powering up, the micro-controller initializes the interfaced sensors and control ports and also verifies every sensor by reading it's hexadecimal identification number. The microcontroller then reads the calibration registers of the BMP180 and MPU-9250 modules to note the initial configuration of the payload and its environment. After completing the initialization and calibration process, the data acquisition process starts by reading the counter ICs on the DAQ system, followed by a read operation of all the peripherals. This operation is repeated every second. The data from two consecutive acquisitions (over a period of two seconds) is stored on registers and then transmitted as a single packet, and is also appended to the on-board EEPROM memory.

The experimental data is transmitted over a serial 3.3V level channel to the telemetry encoder. The packet-length is kept to a maximum of 120 characters with a baud rate of 9600 and refresh rate of $0.5 \mathrm{~Hz}$. The housekeeping data is essential to monitor the health of the payload. This data includes input voltage of High Voltage Power Supply (HVPS), 1000:1 scaled output voltage of HVPS, and positive and negative input voltages to the DAQ system. The housekeeping data is provided as analog buffered voltages to the telemetry encoding system.

\section{Power Supply Systems}

The experimental setup derives power from Lithium-Polymer (Li-Po) batteries. The High Voltage Power Supply (HVPS) is designed to supply power to the PMTs. The HVPS circuit takes in $15 \mathrm{~V}$ DC power as input which is fed to the linear power regulator IC LM7912 which produces an output of $12 \mathrm{~V}$. This voltage is provided to a voltage multiplier, 12AVR1000 by Pico Electronics, which produces an output voltage of $1000 \mathrm{~V}$ DC. The ground-lines of the input and output to the voltage multiplier are kept isolated. The Low Voltage Power Supply (LVPS) board takes in 15V DC and $-9 \mathrm{~V}$ DC supplies as inputs and produces $+5 \mathrm{~V}$ DC, $+3.3 \mathrm{~V}$ DC and $-5 \mathrm{~V}$ DC outputs using linear power-supply-regulator ICs LM7805, LM1117 and LM7905 respectively.

\section{Flight Qualification and Testing}

The experimental setup has been designed to be deployed on a HAB up to $30 \mathrm{~km}$ altitude above mean sea level and hence sustain low pressures of up to $20 \mathrm{mbar}$ and ambient temperatures lower than $-50^{\circ} \mathrm{C}$, during its flight. All the high voltage points have been covered with a silicone elastomer: Sylgard 170, to prevent electric arc discharge. All circuits have been individually enclosed inside aluminum casings to protect from electromagnetic interference (EMI) from external as well as on-board sources. All the interconnections between various subsystems have been routed through D-type high density Amphenol connectors, with redundant connections as precaution. The 
payload has been covered with thermally-insulating polystyrene as a protection from ambient temperatures. The following qualification tests were performed on the experimental payload.

\subsection{PMT Characterization Test}

This test was conducted to determine the optimum operating voltage for the PMTs. The setup includes two qualified detectors with the test detector placed between them, like described in Figure 1; the top and bottom detectors are pre-qualified detectors, while the central detector is the test detector. The two-fold coincidence between the two qualified detectors was compared to the threefold coincidence to determine the efficiency over a range of supply voltage. From Figure 3, we can see that the efficiency for the detector saturates beyond $825 \mathrm{~V}$. Thus, voltage of $1000 \mathrm{~V}$ was chosen as the operating voltage. This test was conducted for all the three PMTs and similar results were observed.

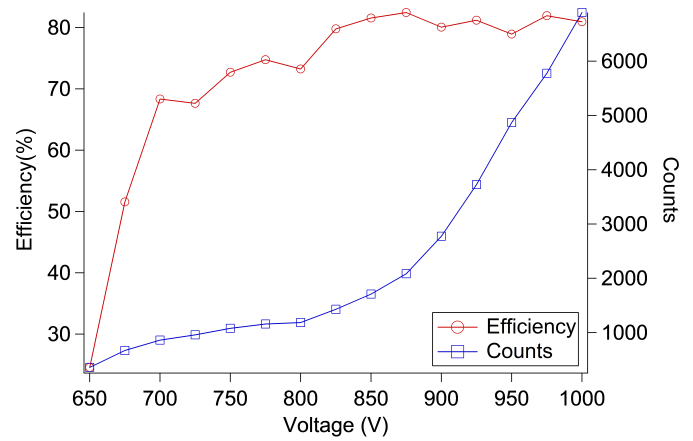

Figure 3: Behavior of PMT1 measurements (in blue) and its efficiency (in orange) as a function of voltage applied at the anode.

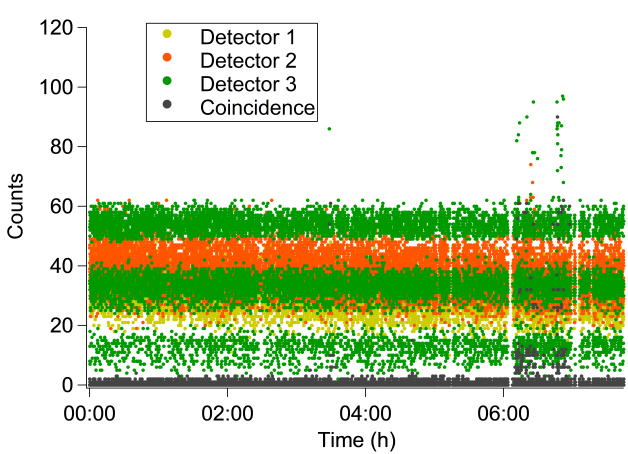

Figure 4: Measurements recorded on the three detector channels and the coincidence channel as a function of time.

\subsection{Long Duration Power-On Test}

The payload's integration with the flight instrument was tested by subjecting the setup to a long duration power-on test wherein it was operated in a room with controlled ambient temperature of about $24^{\circ} \mathrm{C}$ and the data was acquired over telemetry and analyzed for possible errors. This test was conducted for a duration of over 16 hours. Absolute measurements varied (within a satisfactory range) for the detectors, but followed a constant trend in all the three detectors. A few instances have occurred wherein the telemetry sentence has been distorted due to transmission issues, generating erroneous data points, and such points can also be removed from the analysis.

Figure 4, shows a plot of detector measurements against UTC time acquired during this test. It can be observed that all the individual detector counts vary within the same range and the coincidence counts are generally lower than the individual detector counts. The system showed uniformity in operation over an extended duration of time and hence successfully cleared the test.

\subsection{Flight Simulation Test}

The experimental setup was tested in an environmental chamber to simulate the flight conditions. The pressure was reduced to $20 \mathrm{mbar}$, corresponding to an approximate altitude of $30 \mathrm{~km}$ 
from mean sea level. The temperature was then reduced from $28^{\circ} \mathrm{C}$ to $-40^{\circ} \mathrm{C}$ within a span of 70 minutes. These conditions were maintained at these values for 240 minutes. The telemetry digital and analog (consisting of the housekeeping data) channels were monitored for continuity and nominal performance. Temperature probes were mounted on the payload for accurate measurement. A radiosonde was used to measure the ambient temperature and pressure for reference and cross-verification. The test was conducted successfully.

\section{Flight Data: Primary Analysis}

The flight was conducted from the National Balloon Facility in Hyderabad, India using a three thousand cubic metre zero-pressure plastic balloon, and was launched at 2:12 am IST on February 2, 2019. It achieved two float altitudes- $24.8 \mathrm{~km}$ and $26.7 \mathrm{~km}$ above mean sea level. The flight was terminated at 5:17 am and the payload was safely recovered with no visible physical damage and was successfully tested for normal operation, post-flight.

Preliminary analysis of the data recovered from the flight was conducted to ascertain the continuity, quality and accuracy of the data. The first study was to correlate the trend of the altitude measurement from the GPS and the pressure measurement from BMP180; because atmospheric pressure is related to the altitude. Figure 5 illustrates the direct correlation and continuity observed in the measurements from the independent sensors, thereby bringing confidence in the flight data.

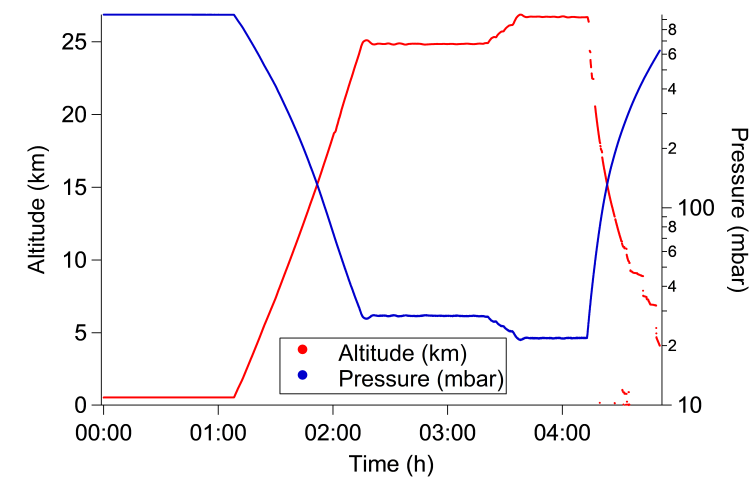

Figure 5: Altitude and Pressure measurements as a function of flight time

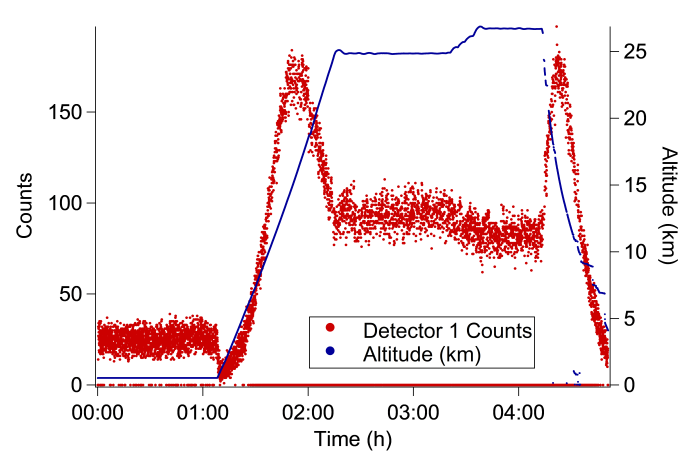

Figure 6: Detector 1 flux and Altitude measurements as a function of flight time

While the individual detector measurements are prone to noise from in-situ radiation and noise, they are nevertheless expected to follow the expected trend of secondary cosmic ray flux as a function of altitude, as stated in [9]. Figure 6 is a plot of the Detector 1 measurements and altitude in the $y$-axis and time in the x-axis. The flux is seen to be rising till the balloon flight reaches an altitude of about $17 \mathrm{~km}$, beyond which the flux starts reducing. The flux measurement stabilizes once the payload reaches its first float altitude and reduces to a second plateau when the payload reaches the second (higher) float altitude. Once the payload starts descending, which is much faster than the ascent, the flux measurement exhibits a similar pattern of a peak at approximately $17 \mathrm{~km}$ altitude. The difference in the ascent and the descent rates can be seen by distinct widths of the flux measurement peaks during the ascent and descent stages. 


\section{Conclusion}

The paper has described the design, construction and qualification tests conducted for an experimental payload to determine cosmic ray flux rates in lower stratospheric regions of the Earth's atmosphere. The payload has been designed to implement a simplistic architecture and use commercially available standard components, while adhering to the standards required to withstand the extreme temperature, pressure and vibration conditions expected during a high-altitude balloon flight. The preliminary analysis of the flight data indicates that the detectors, sensors, flight computers, telemetry and tele-command systems performed nominally thereby confirming the validity and accuracy of the measurements.

\section{Acknowledgements}

The authors are indebted to Tata Institute of Fundamental Research (TIFR) Dept. of High Energy Physics and TIFR Balloon Facility, Hyderabad for the continuous support provided for the HAB flight. The authors acknowledge the support of Project Apeiro team members Pankaj Tiple, Vibhav Joshi and Srihari Menon.

\section{References}

[1] P.L. Biermann, G. Sigl Introduction to cosmic rays. InPhysics and Astrophysics of Ultra-High-Energy Cosmic Rays Springer (pp. 1-26), Berlin, Heidelberg (2001)

[2] J. F. Ziegler, W. A. Lanford The effect of sea level cosmic rays on electronic devices Journal of applied physics, 52(6), 4305-4312 (1981)

[3] J.F. Ziegler The effect of concrete shielding on cosmic ray induced soft fails in electronic systems IEEE Transactions on Electron Devices 28.5, 560-565 (1981)

[4] E. R. Keiter, et al A physics-based device model of transient neutron damage in bipolar junction transistors IEEE Transactions on Nuclear Science 57.6, 3305-3313 (2010)

[5] Chenette, D. L., et al. The CRRES/SPACERAD heavy ion model of the environment (CHIME) for cosmic ray and solar particle effects on electronic and biological systems in space IEEE Transactions on Nuclear Science 41.6, 2332-2339, (1994)

[6] N. K. Belisheva, et al. The effect of cosmic rays on biological systemsâĂŞan investigation during GLE events Astrophysics and Space Sciences Transactions 8.1, 7-17 (2012)

[7] M. K. Lim Cosmic rays: are air crew at risk? Occupational and environmental medicine 59.7, 428-432, (2002)

[8] S. Deshpande, L. N. Kapoor, S. Bheesette and R. R. Shinde, "Embedded system for an airborne payload to determine cosmic ray flux," 2016 IEEE Bombay Section Symposium (IBSS), Baramati, 2016, pp. 1-5, (2016)

[9] F. Lei, et al. An atmospheric radiation model based on response matrices generated by detailed Monte Carlo Simulations of cosmic ray interactions IEEE Transactions on Nuclear Science 51.6. 3442-3451, (2004) 